Document downloaded from:

http://hdl.handle.net/10251/65032

This paper must be cited as:

Brahmi, Y.; Katir, N.; Macia Agullo, JA.; Primo Arnau, AM.; Bousmina, M.; García Gómez, H.; El Kadib, A. (2015). Organophosphonate bridged anatase mesocrystals: low temperature crystallization, thermal growth and hydrogen photo-evolution. Dalton Transactions. 44(35):15544-15556. doi:10.1039/c5dt02367j.

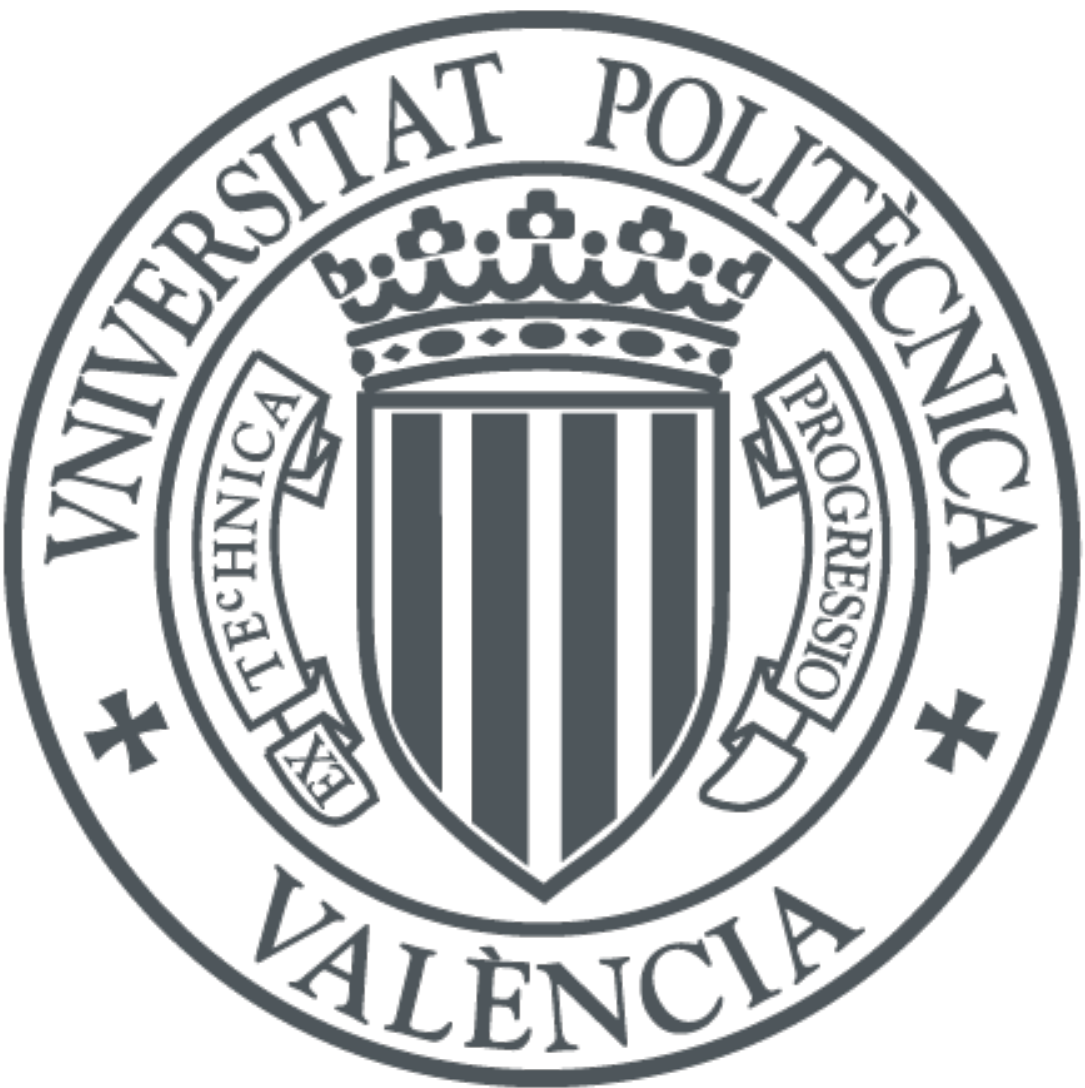

The final publication is available at

http://dx.doi.org/10.1039/c5dt02367j

Copyright Royal Society of Chemistry

Additional Information 


\title{
Organophosphonate Bridged Anatase Mesocrystals: Low Temperature Crystallization, Thermal Growth and Hydrogen Photo-Evolution
}

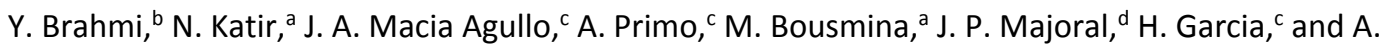 \\ El Kadib*a
}

\begin{abstract}
The sol-gel co-condensation of organo-phosphonate to titanium alkoxide enables access to novel organic-inorganic hybrids based on phosphonate-bridged titanium dioxide. In this contribution, we bring new perspectives to the long established solgel mineralization of titanium alkoxide species, by harnessing the virtues of the well-designed phosphonate-terminated phosphorus dendrimers as reactive amphiphilic nanoreactor, confined medium and cross-linked template to generate at low temperature synthesis $\left(T=60^{\circ} \mathrm{C}\right)$, discrete crystalline anatase nanoparticles. An accurate investigation on several parameters (dendrimer generation, dendrimer-to-titanium alkoxide ratio, precursor reactivity, temperature, solvent nature, salt effect) allows a correlation between the network condensation, the opening porous framework and the crystalline phase formation. The evolution of the dendrimer skeletal upon heat treatment has been deeply monitored by means of ${ }^{31} \mathrm{P} N M R$, XPS and RAMAN spectroscopies. Increasing the heteroatom content within titania network provides the driving force for enhancing their photocatalytic water splitting ability for hydrogen production.
\end{abstract}

\section{Introduction}

The successful coupling of the self-assembly of soft-matter and the sol-gel polymerization of inorganic species, which allow the tight incorporation of organic and inorganic precursors into wellorganized tectonic nanostructures, has been recognized as an historical milestone in the field of functional material synthesis. ${ }^{1}$ Resolving the discrepancy paradigm of soft- and hard- matter chemistry enabled access to stable, texturally-tunable, surfacereactive, multi-functional porous materials. ${ }^{1,2}$ These porous hybrid materials become staple tools in a broad range of applications spanning from adsorption, sensing, catalysis to drug-delivery vehicles and theranostic agents in advanced nanomedicine. ${ }^{2,3}$ Although organosiliceous materials are ubiquitous in this topic, their limited stability issue under hydrothermal or aqueous basic conditions mainly attributed to the intrinsic properties of the silica itself $-^{4}$ raised strong motivations to design more stable hybrid alternatives. ${ }^{5}$ In this framework, the recent trend in hybrid material synthesis marked a substantial shift toward organophosphonate-bridged metal oxides, owing to the greatest stability of P-O-M bonds at the organic-inorganic interface. ${ }^{6,7}$ There are abundant examples concerning the grafting of phosphonic or phosphonate derivatives on metal oxide surface (most of time on $\left.\mathrm{TiO}_{2}\right)^{6}$ and to a less extent, the co-condensation of phosphonate-terminated molecules with soluble titanium alkoxides $\left(\mathrm{Ti}(\mathrm{OR})_{4}\right) .^{7}$ However, these wall-embedded phosphonate-metal oxides are commonly built from amorphous

\footnotetext{
a. Euromed Research Center, Engineering Division, Euro-Mediterranean University of Fes (UEMF), Fès-Shore, Route de Sidi Hrazem, 30070 Fès, Morocco. E-mail: a.elkadib@ueuromed.org

b. Université Mohamed V Agdal, Faculté des Sciences Av. Ibn Battouta, BP 1014 and MAScIR fondation, Rabat, Morocco.

. Instituto de Tecnologia Quimica CSIC-UPV and Departamento de Quimica. Univ. Politicnica de Valencia, Av. de los Naranjos s/n, 46022 Valencia, Spain.

d. Laboratoire de Chimie de Coordination (LCC) CNRS, 205 route de Narbonne,

31077 Toulouse, France.

† Footnotes relating to the title and/or authors should appear here.

Electronic Supplementary Information (ESI) available: [details of any supplementary information available should be included here]. See DOI: $10.1039 / \times 0 \times x 00000 x$
}

titanium dioxide and only few organic-bridged crystalline titania materials have been hitherto disclosed. ${ }^{8}$ This inaccessible class of hybrid crystalline materials obviously outperform its analogue built from amorphous phase as the crystallinity imparts these materials with interesting photo-, opto- and electro-chromic properties. ${ }^{9}$ However, coupling "organophilicity, crystallinity and mesoporosity" within the same tectonic structure is not straightforward and the seeking task faces many difficulties. At low temperature for which the organic part cannot be altered, the inorganic phase is routinely obtained in its amorphous state. ${ }^{10}$ The crystallization - generally operating under thermal treatment - takes place at the expense of the organophilicity and simultaneously induces a dramatic collapse of the opening-framework as a result of the crystal growth. ${ }^{11}$ Recently, various strategies were attempted to reconcile the delicate coupling of "crystallinity-organophilicity" and "crystallinitymesoporosity". For instance, considerable efforts were focused on in situ crystallization of titanium dioxide particles in synthetic liquid medium with the aid of ionic liquids, ${ }^{12}$ benzyl-alcohol ${ }^{13}$ or microwave heating, ${ }^{14}$ using reactive metal chloride or metal fluoride under acidic catalysis $(\mathrm{HCl}$ or even $\mathrm{HF}$ catalyst). While this route proved to be to some extent effective, the use of metal halides and highly corrosive acid catalysts raises questions concerning the protocol safety and its sustainability ${ }^{15}$ Moreover, residual halides - strongly adsorbed to the material surface- have additional drawbacks in electronic and biomedical applications with undesirable effects, pitfalls and misleading results. The simultaneous engineering of the crystallinity and mesoporosity, which is another challenging issue in the chemistry of metal oxides compared to silicates, has been attempted by several approaches. Among the most successful ones, one might cite: i) reinforcing the material walls by carbon-coating and graphitization during thermal treatment to prevent the pore collapsing, ${ }^{16}$ ii) using alternate reactive building-blocks, mainly the so-called "brick and mortar strategy"17 and "nanocrystal-glass approach"18 to adjust the balance between the self-assembly and the wall crystallinity, iii) by charge matching during the early stage of the self-assembly either by heteroatom stabilization ${ }^{19}$ or acid-base pairing ${ }^{20}$ and iv) recently by two-step assembling tectonic nanocrystals in mesoporous network. ${ }^{21}$ 
In this direction, we recently initiated a research program aimed at investigating the beneficial effect of phosphorus-dendrimers to create novel structured mesoporous crystalline materials. ${ }^{7 c-d}$ Our strategy is dictated by several features: i) dendrimers are wellorganized macromolecular species with very intriguing substructure. ${ }^{22}$ ii) They can act as structure directing agents, ${ }^{23}$ confined medium for restricting growth of reactive nano-objects ${ }^{24}$ and as a source of porosity. ${ }^{25}$ All these roles depend on the dendrimer supramolecular chemistry which, in turn, can be engineered on demand. iii) Phosphonate-terminated phosphorus dendrimers are excellent candidates for co-condensation with titanium alkoxide species, thereby ensuring a great stability at the hybrid interface.$^{6-8}$ iv) Besides, the robustness of these functional dendrimers ${ }^{25,26}$ and the presence of a high ratio of nitrogen and phosphorus in their framework make them excellent precursors for heteroatom doping of the resulting titanium dioxide materials. Notably, titania doping with hetero-elements $(N, P, S)$ results in stabilization of the anatase phase and enhancement of its photo-catalytic activity. ${ }^{27}$

In light of these facts, we engaged in-depth studies to rationalize the early crystallization of anatase nanocrystals within the initial tectonic hybrid mesostructures and to accurately assess the consequence of thermal treatment on the crystal growth of the resulting titania. On the whole, these materials are discussed on the basis of the i) low temperature crystallization of titanium oxide species, ii) the porositytype of the as-obtained organic-inorganic tectonic networks and iii) the crystal growth resulting from the thermal annealing treatment. iv) Preliminary results have been also reported concerning the potential use of these materials for hydrogen production.

\section{Results and discussion}

\section{General description of the as-synthesized materials.}

Phosphonate-terminated phosphorus dendrimers were designed by a multistep divergent synthesis starting from the multifunctional hexachlorocyclotriphosphazene $\left(\mathrm{P}_{3} \mathrm{~N}_{3} \mathrm{Cl}_{6}\right)$ that allow the growth of several branching units from the core to the surface (see S1a in Supporting Information for experimental details). For the sake of comparison, a phosphonate branch-mimicking dendrimer (referred as branch) has been obtained from a multistep synthesis procedure (S1a, Supporting Information). The chemical structure of the fourth generation phosphonate-terminated dendrimer $\left(\mathbf{D G}_{\mathbf{4}}\right)$ is shown in Figure 1.

The sol-gel mineralization of titanium alkoxides is a common way to access amorphous titanium dioxide mineral phase. ${ }^{28}$ However, the sol-to-gel transition of titanium-oxo- species is not easy to tune, a fact related to the higher reactivity of hydrolysable titania monomers compared to that of the silica species. ${ }^{4,28}$ Considering this difference, many efforts were oriented to design novel condensable precursors with moderate hydrolysis-condensation reactivity. ${ }^{29}$ For instance, the coordination of titanium to acetylacetonate, carboxylate, phosphonate and pegylated ligands affords novel kineticallycontrolled, titanium-based processable sol-gel building-blocks. ${ }^{30}$ Herein, we revealed that these phosphorus dendrimers play additional roles, first as i) kinetically-modulating precursors that allow to modify the sol-gel polymerization of the initial Ti(OiPr) ${ }_{4}$ solgel precursor, ii) as porous cross-linker reagents that allow to generate a three-dimensional ordered mesoporous network. iii) as reinforcing agents to prevent the collapse of the porosity after thermal annealing treatment and lastly iv) as well-designed precursors for heteroatom (N, $\mathrm{P}$ and $\mathrm{S}$ ) doping of titanium dioxide. The general approach adopted in this work is schematized bellow, illustrating the factors assessed for this mineralization (Scheme 1). Systematically, the sol-gel mineralization is studied as a function of the dendrimer generation, the dendrimer-tometal alkoxide ratio, the solvent nature, the temperature, the precursor reactivity, the salt effect and the reactive-surface located at the dendrimer surface (Table 1 ).

Herein, the standard reaction of the titanium alkoxide mineralization is considered to be: fourth generation phosphonate terminated-phosphorus dendrimers ( $\left.\mathbf{D G}_{4}\right)$, ethanol as solvent, water as co-solvent and catalyst, temperature of $60^{\circ} \mathrm{C}, \mathrm{Ti}(\mathrm{OiPr})_{4}$ as a titania source and a dendrimer-to-metal alkoxide ratio of 1:20 ( $\mathbf{M}_{\mathbf{1}}$ in Table 1$)$. Unless specified, these parameters are kept constant. For example, if the solvent is changed to THF or acetone, the other parameters are those indicated in the standard reaction $\mathbf{D G}_{\mathbf{4}}$, THF or acetone as solvent, water as co-solvent and catalyst, temperature of $60^{\circ} \mathrm{C}, \mathrm{Ti}(\mathrm{O} i \mathrm{Pr})_{4}$ as a titania source and a dendrimer-to-metal alkoxide ratio of 1:20). 


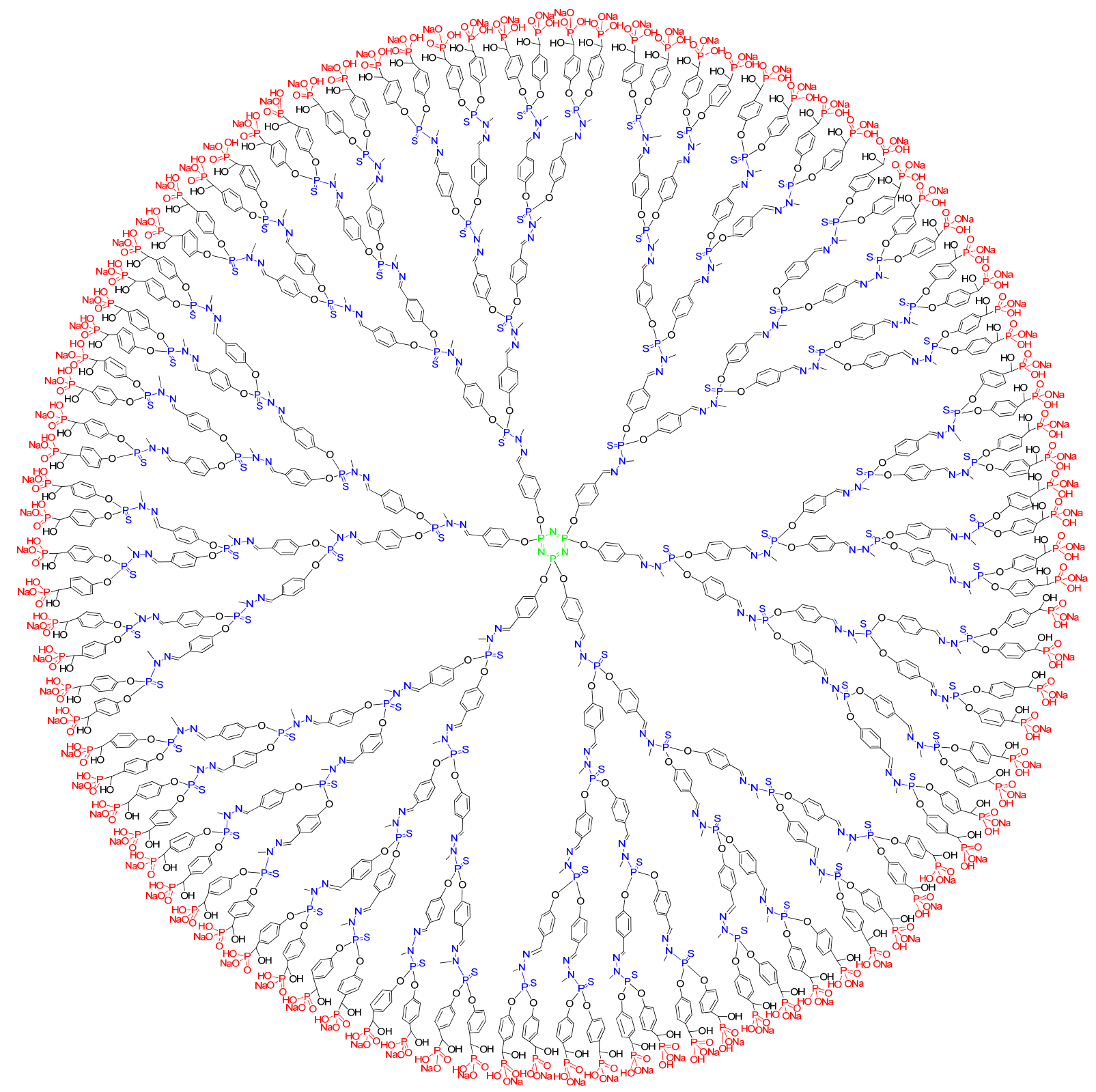

Fig. 1. Structure of the fourth generation phosphonate-terminated dendrimer $\mathbf{D G}_{4}$

Structural composition of the resulting dendrimer-titania solid materials has been systematically elucidated by means of ${ }^{13} \mathrm{C}$ and ${ }^{31} \mathrm{P}$ MAS NMR and DRIFT analysis. In all cases, ${ }^{13} \mathrm{C}$ MAS NMR display signals typical of the parent dendrimers $\left(\mathrm{CH}=\mathrm{NH}, \mathrm{N}-\mathrm{CH}_{3}\right.$ and $\mathrm{CH}-\mathrm{OH}$ resonates at $\sim 137,30$ and $71 \mathrm{ppm}$, respectively and the aromatic rings at 121,131 and $150 \mathrm{ppm}$ ) indicating the preservation of the dendrimer skeletal during titanium alkoxide mineralization (Figure S2, Supporting Information). Of particular interest is the ${ }^{31} \mathrm{P}$ MAS NMR that allows to probe separately the signal of the core ( $\mathrm{P}$ at 6 to $8 \mathrm{ppm}$ ), the branches (at around 62 $\mathrm{ppm}$ ) and those of the surface (at $\sim 16 \mathrm{ppm}$ ) (Figure S2, Supporting Information). Compared to native dendrimers, a slight broadening of the signals assignable to peripheral phosphonates is observed indicating a significant reduction of the molecular mobility at the dendrimer surface because of the expected covalent bonding to the titania network. 


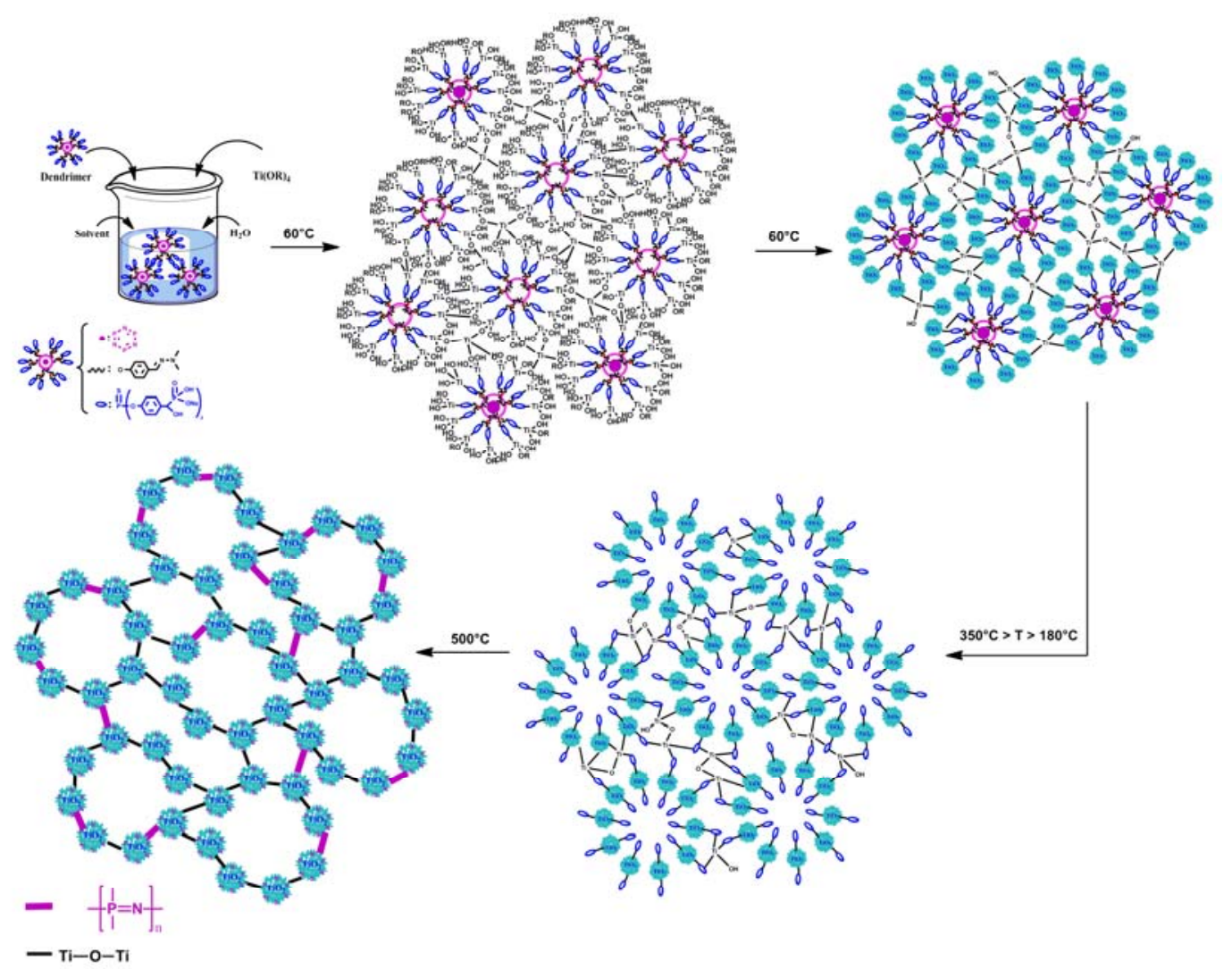

Scheme 1. Multistep synthesis of phosphonate-bridged-anatase mesocrystals. a) The first step consists in the sol-gel chemistry of a titanium precursor (generally Ti(OiPr) 4 ) in the presence of a phosphonate-terminated dendrimer, ethanol as a solvent and water as a co-catalyst at $\mathrm{T}=60^{\circ} \mathrm{C}$. The dendrimer used is built from a repetitive units consisting on an hydrophobic core (cyclophosphazene ring). In each phosphorus of the core grows a branching unit of O-Ph- $\mathrm{C}=\mathrm{N}-\mathrm{N}$. The terminal groups located at the surface consists on $-\mathrm{P}(\mathrm{O})-(\mathrm{OH})-$ $\mathrm{O}^{-} \mathrm{Na}^{+}$. b) The first stage of the sol-gel consists on covalent linkage of phosphonate-terminated dendrimers to titanium alkoxides via P-O-Ti bonds. c) Further nucleation of titanium oxide clusters provides discrete crystalline anatase particles (phosphorus dendrimer plays a role of template to direct the crystal growth of titania nanoparticles). d) Partial destruction of the dendrimer skeletal by ring opening of the core and carbon-heteroatom bond cleavage at the branching points. e) Complete removal of the carbons of the starting dendrimer with the simultaneous heteroatom migration $(\mathrm{N}, \mathrm{P}, \mathrm{S})$ to the titania surface. Adjacent anatase particles are connected either via $\mathrm{P}=\mathrm{N}$ - linkage or Ti-O-Ti bridges.

Table 1. General conditions for the preparation of hybrid dendrimer-titanium dioxide M1 to M15 by sol-gel mineralization process.

\begin{tabular}{|c|c|c|c|c|c|c|c|}
\hline Material & $\begin{array}{l}\text { Titanium } \\
\text { alkoxide }\end{array}$ & Phosphonate $^{a}$ & Ratio (P:Ti) & Solvent & $\mathrm{T}\left({ }^{\circ} \mathrm{C}\right)$ & $\begin{array}{c}\mathrm{S}_{\mathrm{BET}}{ }^{b} \\
\left(\mathrm{~m}^{2} \cdot \mathrm{g}^{-1}\right)\end{array}$ & $\begin{array}{l}\text { Size }^{c} \\
(\mathrm{~nm})\end{array}$ \\
\hline M1 $^{7 \mathrm{c}}$ & $\mathrm{Ti}(\mathrm{OiPr})_{4}$ & $\mathrm{DG}_{4}$ & $1: 20$ & EtOH & 60 & 203 & 4.8 \\
\hline $\mathbf{M}^{7 \mathrm{c}}$ & $\mathrm{Ti}(\mathrm{OiPr})_{4}$ & $\mathrm{DG}_{3}$ & $1: 20$ & $\mathrm{EtOH}$ & 60 & 291 & 5.8 \\
\hline $\mathrm{M}^{7 \mathrm{c}}$ & $\mathrm{Ti}(\mathrm{OiPr})_{4}$ & $\mathrm{DG}_{2}$ & $1: 20$ & $\mathrm{EtOH}$ & 60 & 386 & 6 \\
\hline M4 & $\mathrm{Ti}(\mathrm{OiPr})_{4}$ & Branch & $1: 20$ & $\mathrm{EtOH}$ & 60 & 376 & $-d$ \\
\hline M5 & $\mathrm{Ti}(\mathrm{OiPr})_{4}$ & ------ & ---- & $\mathrm{EtOH}$ & 60 & 384 & $-d$ \\
\hline M6 & $\mathrm{Ti}(\mathrm{OiPr})_{4}$ & $\mathrm{DG}_{4}$ & $1: 5$ & $\mathrm{EtOH}$ & 60 & 456 & 5.7 \\
\hline M7 & $\mathrm{Ti}(\mathrm{OiPr})_{4}$ & $\mathrm{DG}_{4}$ & $1: 10$ & $\mathrm{EtOH}$ & 60 & 361 & 6.5 \\
\hline M8 & $\mathrm{Ti}(\mathrm{OtBu})_{4}$ & $\mathrm{DG}_{4}$ & $1: 20$ & $\mathrm{EtOH}$ & 60 & 172 & 4.5 \\
\hline M9 & $\mathrm{Ti}(\mathrm{OiPr})_{2}(\mathrm{acac})_{2}$ & $\mathrm{DG}_{4}$ & $1: 20$ & $\mathrm{EtOH}$ & 60 & 158 & $-d$ \\
\hline M10 & $\mathrm{Ti}(\mathrm{OiPr})_{4}$ & $\mathrm{DG}_{4}$ & $1: 20$ & $\mathrm{EtOH}$ & 100 & 232 & 5.8 \\
\hline M11 & $\mathrm{Ti}(\mathrm{OiPr})_{4}$ & $\mathrm{DG}_{4}$ & $1: 20$ & $\mathrm{EtOH}$ & 25 & 28 & -d $^{-1}$ \\
\hline M12 & $\mathrm{Ti}(\mathrm{OiPr})_{4}$ & $\mathrm{DG}_{4}$ & $1: 20$ & $\mathrm{EtOH}$ & U-sounde & 382 & $-{ }^{d}$ \\
\hline M13 & $\mathrm{Ti}(\mathrm{OiPr})_{4}$ & $\mathrm{DG}_{4}$ & $1: 20$ & Acetone & 60 & 159 & 4.6 \\
\hline M14 & $\mathrm{Ti}(\mathrm{OiPr})_{4}$ & $\mathrm{DG}_{4}$ & $1: 20$ & THF & 60 & 32 & 4.9 \\
\hline M15 & $\mathrm{Ti}(\mathrm{OiPr})_{4}$ & $\mathrm{D}-\mathrm{G}_{2} \mathrm{POOMe}^{f}$ & $1: 20$ & $\mathrm{EtOH}$ & 60 & 127 & 4.7 \\
\hline
\end{tabular}

${ }^{a}$ the organo-terminated phosphonates additives introduced in the medium. $\mathbf{D G}_{4}, \mathbf{D G}_{3}$ and $\mathbf{D G}_{2}$ are respectively the fourth, third and second generation phosphonateterminated dendrimers $\left(\left(\mathrm{P}(\mathrm{O})(\mathrm{OH})\left(\mathrm{O}^{-} \mathrm{Na}^{+}\right)\right)\right.$. The branch is referred to a single molecule with similar external surface of the used dendrimers . The exact chemical structure of each building-block is shown in the supplementary information. ${ }^{b}$ Specific surface areas of the as-synthesized hybrid materials as determined by nitrogen sorption analysis. ${ }^{c}$ the particle size of the crystalline anatase calculated from X-ray diffraction analysis (for the as-synthesized materials). ${ }^{d}$ Titanium dioxide is obtained in its amorphous state and no crystalline peaks have been detected. ${ }^{e}$ Ultrasonication for 2 hours where the starting temperature is $25^{\circ} \mathrm{C}$ and reaches $45^{\circ} \mathrm{C}$ within the two hours. ${ }^{f}$ The terminal phosphonate of this second generation dendrimer is $\mathrm{P}(\mathrm{O})(\mathrm{OMe})_{2}$ rather than $\mathrm{P}(\mathrm{O})(\mathrm{OH})\left(\mathrm{O}^{-} \mathrm{Na} a^{+}\right.$.

SEM analysis reveals the presence of homogeneous microspheres built from the co-condensation of various elementary dendritic building blocks and titanium oxide clusters with no over-aggregated, phase-separation or uncontrolled growth of the reactive titanium oxide-based oligomeric species (Figure 2a). 


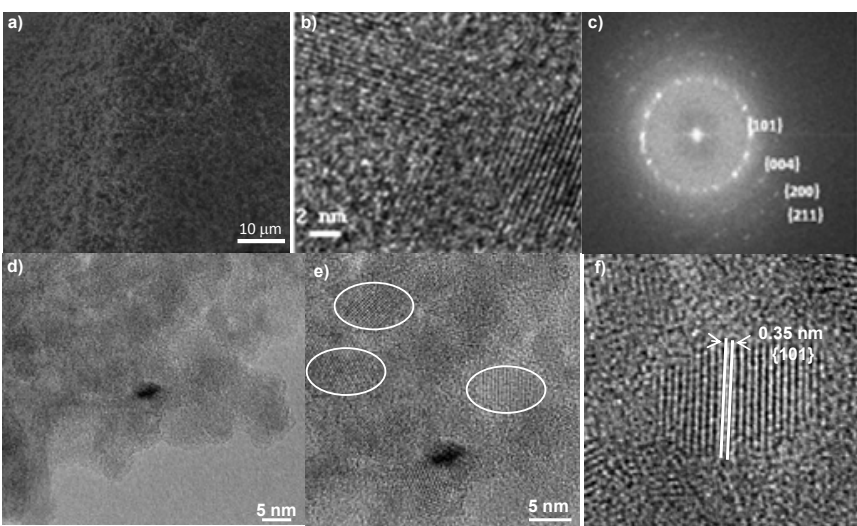

Fig. 2. Microscopic characterization of the as-synthesized dendrimer-titanium dioxide hybrid materials. a) SEM photo of $\mathbf{M}_{\mathbf{3}}$. b) HRTEM of $\mathbf{M}_{\mathbf{3}}$. c) SAED of $\mathbf{M}_{\mathbf{3}}$ showing crystalline plane of anatase nanoparticles. d, e) HRTEM of $\mathbf{M}_{1}$ showing discrete anatase (inside of the cerles) entangled in a mesoporous network. f) Lattice fringe of the crystal showing the typical 101 plan of the anatase phase.

\section{Low temperature crystallization, mesoporosity and stability}

Influence of the dendrimer generation on titanium alkoxide mineralization:

"Dendrimer effect", the specific behavior of a given generation compared to its sisters or even to the parent, isolated monomer is a well-known phenomenon that has been elucidated in catalysis and biology. ${ }^{31}$ Following our involvement in material design by dendrimer approach, $7,25,32$ the first question raised is: is there any difference in templating the mineralization of titanium alkoxide with different dendrimer's generation? To address this question, second, third and fourth generation phosphonate-terminated phosphorus dendrimers $\left(\mathbf{D G}_{n}, n=2,3\right.$ and 4) were co-condensed with $\mathrm{Ti}(\mathrm{OiPr})_{4}\left(\mathbf{D G}_{\mathrm{n}}: \mathrm{Ti}\right.$ ratio $\left.=1: 20\right)$ in $\mathrm{EtOH} / \mathrm{H}_{2} \mathrm{O}$ for 10 hours at $60^{\circ} \mathrm{C}$ to afford $\mathbf{M}_{\mathbf{3}}, \mathbf{M}_{\mathbf{2}}$ and $\mathbf{M}_{\mathbf{1}}$, respectively (See Table 1 and the Experimental section for details). Whatever the generation used, $\mathrm{X}$-ray diffraction shows delightedly the successful formation of crystalline peaks unambiguously assignable to the anatase phase of titanium dioxide (Figure S3, Supporting Information). The average size of the nanocrystals calculated from the half-height width of the [101] peak using the Scherrer formula ranges from 4.8 to $6 \mathrm{~nm}$ (Figure 3a). HRTEM and SAED corroborated these results as small, discrete nanoparticles are shown to be well-entangled within the dendritic-titania networks. At high magnification, individual crystals of $\sim 5 \mathrm{~nm}$ with interplanar distance of $3.5 \AA$ are observed, in superb accordance with $\mathrm{X}$-ray diffraction analysis (Figure $2 b-f$ and Figure S4, Supporting Information). In parallel, nitrogen sorption analysis evidenced an open, mesoporous network with specific surface areas ranging from 203 to $386 \mathrm{~m}^{2} \cdot \mathrm{g}^{-1}$ (Figure $3 \mathrm{~b}$ ). Notably, increasing the dendrimer generation induces significant decrease of both the crystal size and the specific surface area. In contrast, when single branch terminated phosphonate co-condense to metal alkoxide in similar way to dendrimer-metal alkoxide (preparation of $\mathbf{M}_{\mathbf{4}}$ ), the mineralization affords only amorphous titanium dioxide (Figure 3a). Similarly to this branch-mimicking dendrimer, the blank experiment - conducted for the preparation of $\mathbf{M}_{\mathbf{5}}$ in the absence of any phosphonate additive - failed also to afford crystalline phase (Figure 3a). These results unambiguously substantiate the "particularism" of the phosphorus dendrimers (versus monomers) during titanium alkoxide sol-gel mineralization.
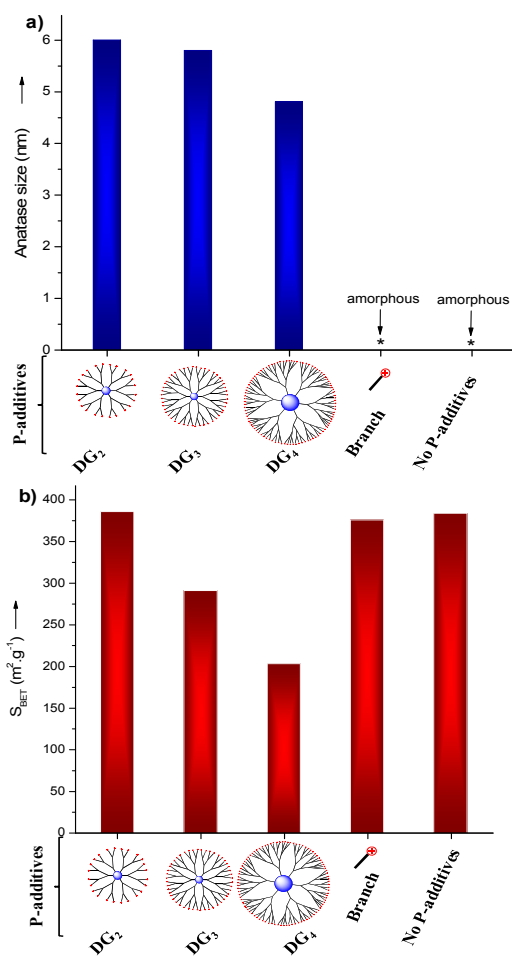

Fig. 3. Sol-gel mineralization of titanium tetra-isopropoxide in ethanol-water mixture at $60^{\circ} \mathrm{C}$ performed in the presence of, from left to right: $\mathrm{DG}_{2}, \mathrm{DG}_{3}, \mathrm{DG}_{4}$, the branchmimicking dendrimer and lastly, without any phosphorus additive. a) Crystal size estimation by X-ray diffraction (from Scherrer formula). b) Specific surface area measurements by nitrogen physisorption.

\section{Influence of the dendrimer: titanium alkoxide ratio:}

Having substantiated the pivotal role of phosphorus dendrimers in the formation of crystalline particles at low temperature, we turned our attention to examine the influence of the functional phosphonate-to-metal alkoxide ratio. With this aim, three experiments were conducted for each dendrimer generation with a dendrimer-to-metal alkoxide ratio of 1:5, 1:10 and 1:20 (the ratio is calculated basing on the number of the functional group located at the periphery) to afford in the case of the fourth generation $\mathbf{M}_{\mathbf{6}}, \mathbf{M}_{\mathbf{7}}$ and $\mathbf{M}_{\mathbf{1}}$, respectively (Table 1 ). While the dendrimer-to-metal alkoxide ratio does not seem to disrupt neither the crystalline phase formation, nor the crystal size (the anatase size varies from 4.5 to $8 \mathrm{~nm}$ with no clear trend), nitrogen sorption reveals a certain dependency on the used ratio (Figure 4 and Figure S5, Supporting Information). Indeed, the dendrimer-anatase hybrids synthesized with a 1:5 ratio display an isotherm profile typical of super-microporous materials with a significant amount of microporosity and specific surface areas ranging from 456 to $511 \mathrm{~m}^{2} \cdot \mathrm{g}^{-1}$. In contrast, rising the ratio to $1: 10$ and then to $1: 20$ seems to be beneficial for the creation of a disordered porous network as evidenced by the appearance of hysteresis loop that is indicative of capillary condensation within the mesopores. The dendrimer-to-metal alkoxide ratio has indeed an effect of the textural properties, allowing the adjustment of the micro-, meso- and interporosity of the resulting hybrid dendrimertitanium dioxide materials. 


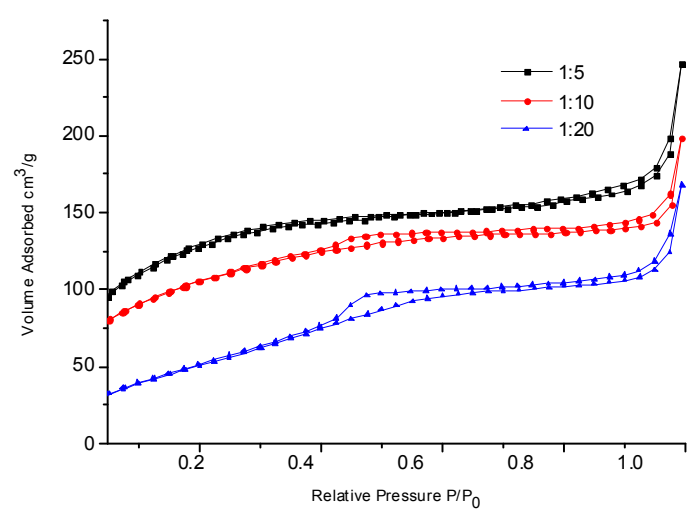

Fig. 4. Nitrogen sorption isotherm profile of the as-synthesized dendrimer-titanium dioxide with different phosphonate-to-Ti ratio (1:5, 1:10 and 1:20). These materials are respectively referred in table 1 as $\mathbf{M 6}, \mathbf{M 7}$ and $\mathbf{M} \mathbf{1}$

Moreover, substantial difference in their stability has been revealed by thermo-gravimetric analysis (TGA). Expectedly, the amount of degraded materials (at $800^{\circ} \mathrm{C}$ ) decreases with increasing the dendrimer-to-metal alkoxide ratio (from 1:5 to 1:20) (Figure S6, Supporting Information). As exemplified for the second generation $\left(\mathbf{D G}_{\mathbf{2}}\right), 30 \%$ of the weight loss is observed for the material prepared with $1: 5$ ratio, $20 \%$ for $1: 10$ and $15 \%$ when using a ratio of 1:20. Similar trend is observed for the third $\left(\mathbf{D G}_{3}\right)$ and the fifth generation $\left(\mathbf{D G}_{4}\right)$ dendrimers, respectively (22\% and $28 \%$ for $1: 5 ; 17 \%$ and $19 \%$ for $1: 10$ and only $15 \%$ for 1:5). The weight loss occurs mainly at temperatures below $200^{\circ} \mathrm{C}$, indicating the presence of a significant amount of water as well as strongly physisorbed ethanol and residual alkoxides from the incomplete condensation of titanium species. The densification of the network occurs above $200^{\circ} \mathrm{C}$ by reacting Ti$\mathrm{OH}$ (and $\mathrm{Ti}-\mathrm{OR}$ ) to each other to create more robust Ti-O-Ti network.

Influence of the monomer hydrolysis-condensation kinetics:

To gain a better understanding of the kinetically controlled solgel process in presence of these phosphonate-terminated
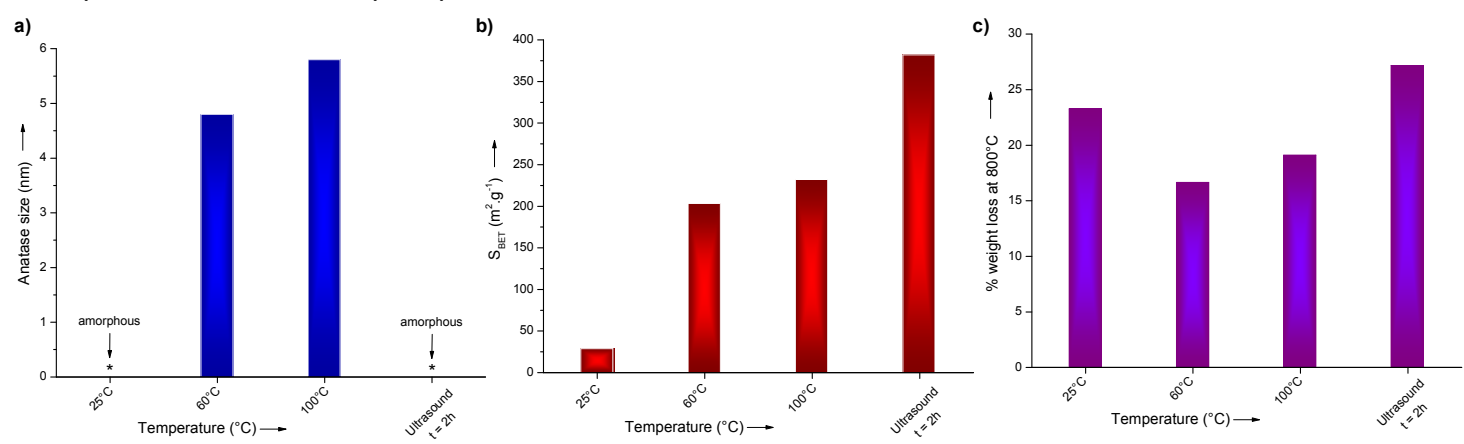

Fig. 5. Sol-gel mineralization of titanium tetra-isopropoxide, ethanol-water performed in presence of $\mathbf{D G}_{4}$ at, from left to right: $25^{\circ} \mathrm{C}, 60^{\circ} \mathrm{C}, 100^{\circ} \mathrm{C}$ (for $10 \mathrm{~h}$ ) and lastly under sonication for $2 \mathrm{~h}$. These materials are respectively referred in Table 1 as $\mathbf{M}_{11}, \mathbf{M}_{\mathbf{1}}, \mathbf{M}_{10}$ and $\mathbf{M}_{\mathbf{1 2}}$ : a) Crystal size estimation by $\mathbf{X}$-ray diffraction (from Scherrer formula). b) Specific surface area measurements by nitrogen physisorption. c) Percents of weight loss calculated by TGA at $800^{\circ} \mathrm{C}$.

whereas the bulky $\mathrm{Ti}(\mathrm{O} t \mathrm{Bu})_{4}$ slightly reduces the open porous network. $\mathrm{Ti}(\mathrm{acac})_{2}(\mathrm{OiPr})_{2}$ in consistency with its weak condensation exhibits the lowest specific surface area with an isotherm profile being more characteristic of polymeric, microporous network.

\section{Influence of the Temperature $\left(T=25^{\circ} \mathrm{C}, 60^{\circ} \mathrm{C}\right.$ and $\left.100^{\circ} \mathrm{C}\right)$ :}

We further investigated the consequence of varying the sol-gel temperature on the resulting mineral phase, the framework dendrimers, mineralisation of titanium species has been accomplished using three different alkoxides, namely : $\mathrm{Ti}(\mathrm{O} i \mathrm{Pr})_{4}$, $\mathrm{Ti}(\mathrm{OtBu})_{4}$ and $\mathrm{Ti}(\mathrm{OiPr})_{2}(\mathrm{acac})_{2}$. While the three precursors are prone to hydrolysis-condensation under sol-gel polymerization chemistry, their ability to form -(Ti-O-Ti) ${ }_{n}$ nano-sized clusters is ligand-dependent, with decreasing the nucleation kinetics from iso-propoxide to tert-butoxide to the more persistent acetylacetonate groups. ${ }^{33}$ We were therefore interrogated if low temperature crystallization, observed at $60^{\circ} \mathrm{C}$, is a specific phenomenon induced by the dendrimer environment (possible confinement effect, specific interface properties, ect) or a kinetically controlled process that require an energy input to provide crystalline phase upon hydrolysis-condensation. After 10 hours at $60^{\circ} \mathrm{C}$, the isolated solid materials were analyzed by XRD. Interestingly, the two materials $\left(\mathbf{M}_{\mathbf{1}}\right.$ and $\left.\mathbf{M}_{\mathbf{8}}\right)$ obtained from $\mathrm{Ti}(\mathrm{O} i \mathrm{Pr})_{4}$ and $\mathrm{Ti}(\mathrm{OtBu})_{4}$ as titania sources afford crystalline anatase with an average size of 4.8 and $4.5 \mathrm{~nm}$, respectively. By sharp contrast, the less-reactive $\mathrm{Ti}(\mathrm{O} i \mathrm{Pr})_{2}(\mathrm{acac})_{2}$ failed to generate crystalline anatase and only amorphous, mineral titanium dioxide were obtained in $\mathbf{M}_{\mathbf{g}}$. Indeed, while the presence of phosphonate-terminated phosphorus dendrimers is mandatory for the formation of discrete crystalline particles, an activation energy barrier has to be surmounted (most probably using kinetically reactive sol-gel species) for providing crystalline phase.

This precursor variation has also a dramatic influence on the material stability as illustrated during thermal degradation. Although the same dendrimer: metal alkoxide ratio $(1: 20)$ is used for the three materials, their weight loss varies significantly. The material degradation profile seems in perfect accordance with the predictable sol-gel kinetics; the increased amount of weight loss being attributed to residual incondensable fragments (acac)OiPr>OtBu>OiPr (Figure S7, Supporting Information). The highest specific surface area is obtained for the hybrid starting from $\mathrm{Ti}_{(\mathrm{O} i \mathrm{Pr})_{4}}$ as precursor, 
energy input, even though $\mathrm{Ti}(\mathrm{O} i \mathrm{Pr})_{4}$ is generally considered as a reactive hydrolysable monomer. Second, the resulting material features only marginal specific surface area of $28 \mathrm{~m}^{2} \cdot \mathrm{g}^{-1}$. The dendrimer topology is slightly temperature-sensitive (in terms of swelling-subsiding) being even at the basis of their use as hosts for smaller objects and for the encapsulation of biomolecules. ${ }^{34}$ This characteristic may explain to some extent the discrimination seen herein for the resulting porous network. Extended reaction time is also revealed of importance $(t=10 \mathrm{~h})$, since the ultra-sonication for only two hours - supposed to bring the necessary energy for crystallization - did not induce the expected anatase formation (preparation of $\mathbf{M}_{\mathbf{1 2}}$ ). Therma properties of these hybrid materials were found to be temperature-dependent; a fact attributed to the weakness of the cross-linked network both at $25^{\circ} \mathrm{C}$ and under sonication compared to the significant condensation occurring for the materials prepared at $60^{\circ} \mathrm{C}$ and $100^{\circ} \mathrm{C}$ (Figure S8, Supporting Information).

\section{Solvent effect:}

The solvent nature (polarity, lipophilicity, hydrophobicity...) is well known to affect the textural properties of the kinetically controlled sol-gel of siliceous materials. ${ }^{35}$ No report has addressed, however, its probable implication on selective crystalline-phase formation for non-siliceous metal oxide. Accordingly, we decided to devise answers for the involvement of at least three different solvents in promoting crystalline anatase versus amorphous titania. For reasons of dendrimer solubility (thereby assessing only crystallization occurring under homogeneous conditions), the choice of the solvents was limited to ethanol, acetone and tetrahydrofurane (THF) (preparation of $\mathbf{M}_{\mathbf{1}}, \mathbf{M}_{\mathbf{1 3}}$ and $\mathbf{M}_{\mathbf{1 4}}$, Table 1). Whatever the solvent used, $\mathrm{Ti}(\mathrm{OiPr})_{4}$ mineralization enabled to obtain, at $60^{\circ} \mathrm{C}$, discrete crystalline anatase with crystal size ranging from 4.6 to $4.9 \mathrm{~nm}$. The openness of their framework seems, however, solvent-sensitive with THF leading to a marginal specific surface area of $32 \mathrm{~m}^{2} \cdot \mathrm{g}^{-1}$, compared to that observed for acetone and ethanol (159 and $203 \mathrm{~m}^{2} \cdot \mathrm{g}^{-1}$ respectively). The highest condensation degree seems to be attained with ethanol; a fact that is attributed to the fast alkoxy- exchange at the titania center, while less cross-linked network is induced by the use of bulky THF solvent (TGA profile, See Figure S9, Supporting Information).

\section{Salt's effect:}

To glean a broad understanding on the global parameters suspected to be involved in the crystal formation at relatively low temperature and its stability at high temperature, the role of the functional groups located at the dendrimers surface has been accurately examined. Notably, the external function on the dendrimer-surface governs the early stage of the dendrimer ---> titanium alkoxide interactions to provide novel modified sol-gel precursors (kinetic effect) and influences the stability at the organic-inorganic interface (thermodynamic effect). ${ }^{7 \mathrm{c}} \mathrm{We}$ therefore decided to enlarge the library of the reactive-surface dendrimers for titanium dioxide mineralization $\left(\mathbf{M}_{\mathbf{3}}\right.$ and $\mathbf{M}_{\mathbf{1 5}}$ Table 1). Hence, phosphonate salt $-\mathrm{PO}(\mathrm{OH}) \mathrm{O}^{-}, \mathrm{Na}^{+}$versus phosphonate diesters - $\mathrm{PO}(\mathrm{OMe})_{2}$ decorated second generation phosphorus dendrimers $\left(G_{2}\right)$ were compared for $\mathrm{Ti}(\mathrm{OiPr})_{4}$ mineralization. This slight modification has been motivated by the beneficial role of the salt-effect on tuning the silica mesostructure ${ }^{36}$ and for its inhibitory action against the crystal growth. ${ }^{37}$ In principle, no difference in the crystallization phenomenon was observed since the two materials provide discrete crystalline anatase ( 4.7 and $6 \mathrm{~nm}$ in size). However, the salt-effect is found to be useful to enhance the specific surface area, being $386 \mathrm{~m}^{2} \cdot \mathrm{g}^{-1}$ for $\mathbf{M}_{\mathbf{3}}$ obtained from - $\mathrm{PO}(\mathrm{OH}) \mathrm{O}^{-}, \mathrm{Na}^{+}$ compared to $127 \mathrm{~m}^{2} \cdot \mathrm{g}^{-1}$ for $\mathbf{M}_{15}$ obtained from -PO(OMe) terminated phosphorus dendrimer.

\section{Evolution of the chemical structure by thermal annealing of the} hybrids

With these novel nanostructures in hand, the issues to be next addressed were: how the mineral phase responds to the thermal activation? and what will be the destiny of these robust dendrimers within the material framework? Crystallization of the amorphous phase and expansion of the crystal size are the most frequent scenarios induced in the bulk by thermal annealing. ${ }^{11}$ To get more insight, thermal evolution of these microstructures has been first monitored by solid-state NMR spectroscopy. ${ }^{13} \mathrm{C}$ and ${ }^{31} \mathrm{P}$ MAS NMR of the materials resulting from calcination at 180,350 and $500^{\circ} \mathrm{C}$ were recorded (Figure $6 a, b)$. As expected, carbon-carbon and carbon-heteroatom bonds cleavage $(\mathrm{C}=\mathrm{N} ; \mathrm{N}-\mathrm{Me}$ and $\mathrm{CH}-\mathrm{OH})$ take place upon heat treatment at $180^{\circ} \mathrm{C}$, slight graphitization (persistence of the aromatics) is observed at $350^{\circ} \mathrm{C}$ and lastly, complete loss of the carbon signal occurs at $500^{\circ} \mathrm{C}$ (Figure 6a). In parallel, significant reduction of the phosphorus signal located at the periphery is observed upon heat treatment at $180^{\circ} \mathrm{C}$ with consequent apparition of a new one at $-6 \mathrm{ppm}$ (Figure 6b). Thermal annealing at $350^{\circ} \mathrm{C}$ resulted in complete loss of the phosphorus located at the branching position as well as those of the core, whereas only one broad signal is observed at around $-6 \mathrm{ppm}$ (Figure 6b). The latter can be tentatively attributed to a tetrahedral phosphorus environment bridged to the titania center $(\mathrm{Ti}-\mathrm{O}-\mathrm{P})_{\mathrm{x}}{ }^{38}$ This seems to indicate a migration of the phosphorus units (those of the surface first and then, those of the branch and the core) to the anatase particle surface probably by reaction with residual $\mathrm{Ti}-\mathrm{OH}$ to form Ti-O-P. This can explain to some extent the presence of a unique broad phosphorus signal within the thermally-annealed material. CHN elemental analysis indicated that $98 \%$ of $\mathrm{C}$ atoms and up to $85 \%$ $\mathrm{H}$ atoms are removed at $500^{\circ} \mathrm{C}$ while $\mathrm{N}, \mathrm{S}$ and $\mathrm{P}$ remains unaltered after calcination. Considering the high portion of $P, N$ and $\mathrm{S}$ heteroatoms in the starting dendrimers, this further explains the lower weight loss (less than 15 to 20\%) observed for most of these hybrid materials.

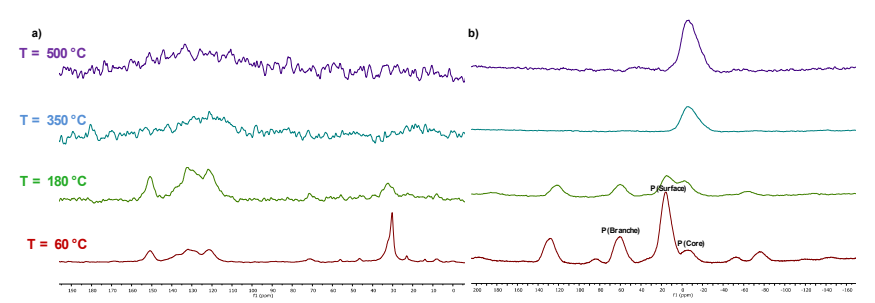

Fig. 6. Solid state NMR of the hybrid dendrimer-titania materials recorded at $60^{\circ} \mathrm{C}, 180^{\circ} \mathrm{C}, 350^{\circ} \mathrm{C}$ and at $500^{\circ} \mathrm{C}$. a) ${ }^{13} \mathrm{C}$ MAS NMR and b) ${ }^{31} \mathrm{P}$ MAS NMR.

Further understanding has been gained from XPS analysis by comparing hybrid dendritic-titania materials before and after thermal annealing. The as-synthesized dendrimer-titania hybrid exhibits signals with binding energy characteristic of titanium 
dioxide (461.48 for Ti2 $\mathrm{p}_{3 / 2}$ and $467.38 \mathrm{eV}$ for Ti2 $\mathrm{p}_{1 / 2}$ ) (figure 7a). One broad signal is observed for $\mathrm{P} 2 \mathrm{p}$ at $136.12 \mathrm{eV}$ due to the over-lap of the binding energy of phosphorus belonging to the surface (phosphonate) and the one located at the core (cyclophosphazene) (figure 7b).

Three different nitrogen species are also detected at 394.23, 397.93 and $402.63 \mathrm{eV}$ (figure 7c). Exceeding a binding energy value of $401 \mathrm{eV}$ indicates the quaternization of nitrogen element probably by donation to the acidic titania centers ( $\mathrm{N}$--$>$ Ti). ${ }^{33 \mathrm{~d}}$ Upon calcination, the persistence of phosphorus and nitrogen within the microstructure has been corroborated by the observation of a broad signal at a binding energy of 136.9 for phosphorus eV and at 394.42, 398.42 and $403.72 \mathrm{eV}$ for nitrogen species (figure $7 \mathrm{e}, \mathrm{f}$ ). The signal of titanium dioxide appears at 462.88 and $468.38 \mathrm{eV}$ (figure $7 \mathrm{~d}$ ). Similar chemical shifts to higher binding energy upon thermal annealing has been already reported in the case of hypophosphorus doped titania and has been attributed to the formation of Ti-O-P within the anatase lattice. ${ }^{39}$ Taking also into account the possibility for cyclotriphosphazene ring opening upon heat treatment, ${ }^{7 d, 38 c}$
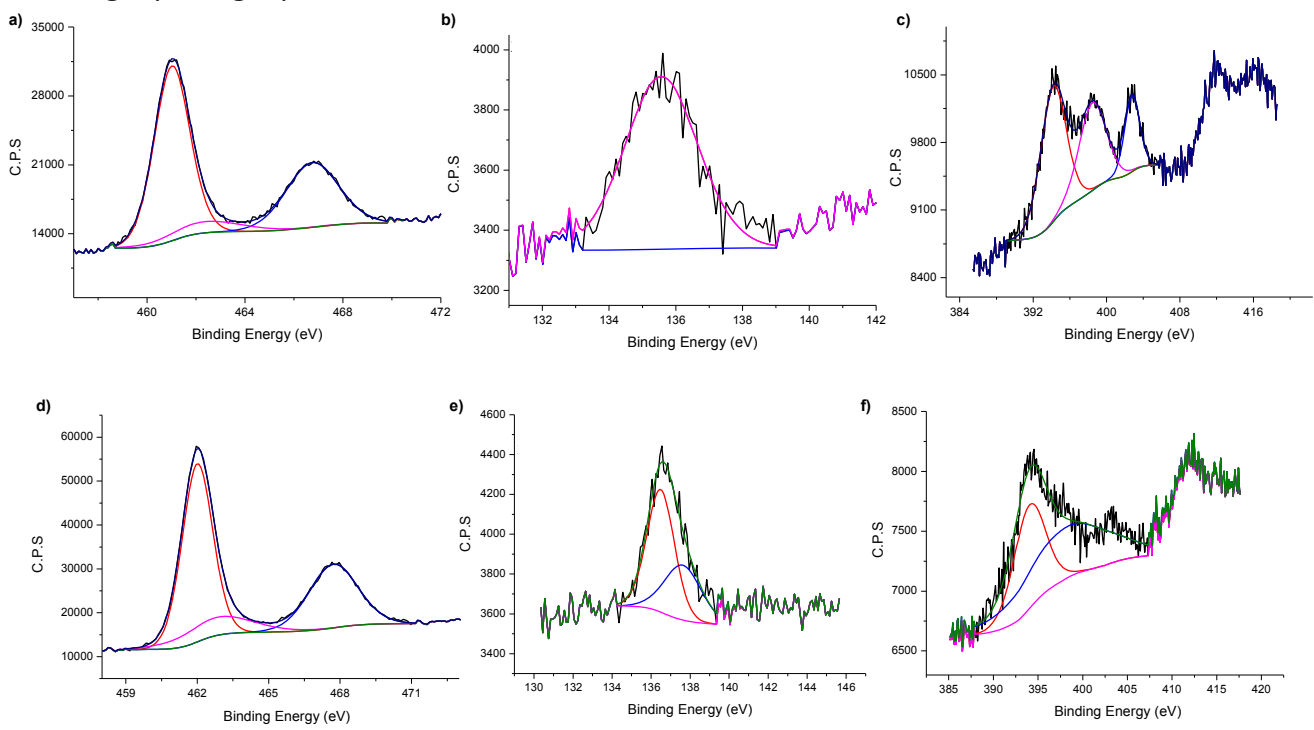

Fig. 7. XPS analysis of the isolated dendrimer-titanium dioxide hybrid materials a-c) before and d-f) after thermal annealing. From right to left: the binding energy of $\mathrm{Ti}, \mathrm{P}$ and $\mathrm{N}$.

In contrast, standard materials ( $\mathbf{M}_{\mathbf{1}}$ as an example) display four peaks at values of $144,395,514$ and $639 \mathrm{~cm}^{-1}$, characteristic of crystalline anatase $\mathrm{e}^{39,40}$ (Figure 8a). However, mapping in various regions reveals in some cases the predominance of amorphous titania species and/or the scarcity of crystalline anatase. Upon calcination, homogeneous, well-resolved, crystalline anatase is obtained as illustrated in Figure $8 \mathrm{~b}$. This result indicates that the as-synthesized material are built from a mixture of amorphous titanium dioxide and discrete crystalline anatase phase. Thermal treatment improves the crystallinity of the material framework much probably by converting those initially obtained as amorphous phase to their crystalline state.

UV-visible spectroscopic studies performed on the solid materials (before and after thermal treatment) indicate the presence of a large band with a maximum at $\sim 324 \mathrm{~nm}$ which is consistent with an incipient oligomerization of $\mathrm{Ti}(\mathrm{IV})$ species (Figure 8c). However, the as-synthesized materials adsorb in the visible region (solids with beige color), while the thermally end-capping of residual titanols (Ti-OH) and lastly, the fusion and migratory insertion of the heteroatoms within the surface of anatase nanocrystals, a variety of heteroatom doping molecular species can be suggested (-Ti-O-P-, -Ti-O-N-, - N=P-O$\mathrm{Ti}-$ ) in consistency with the conclusions drawn from solid state NMR analysis. While discrete crystalline anatase particles are observed at $60^{\circ} \mathrm{C}$ for most of these dendrimer-titania materials (except those performed with $\mathrm{Ti}(\mathrm{acac})_{2}(\mathrm{OiPr})_{2}$ or at $\mathrm{T}$ of $25^{\circ} \mathrm{C}$ ) (See $\mathbf{M}_{\mathbf{9}}, \mathbf{M}_{\mathbf{1 1}}$ and $\mathbf{M}_{\mathbf{1 2}}$ in Table 1 ), this fact does not exclude the presence of amorphous titania units, specifically those directly bridged to the dendrimer surface. To get insight on this and to further assess the evolution of the mineral phase after thermal treatment, RAMAN spectroscopic studies were performed on a presentative materials before and after calcination. To avoid any misleading interpretation, fully amorphous materials were first assessed. Indeed, the material $\mathbf{M}_{\mathbf{g}}$ drived from the mineralization of $\mathrm{Ti}(\mathrm{acac})_{2}(\mathrm{OiPr})_{2}$ and $\mathbf{M}_{\mathbf{4}}$ performed by branchmimicking dendrimers do not reveal any characteristic signal of crystalline titania (Figure S10, Supporting Information). 
boundaries of the crystal grains constitutes the driving force to prevent the pore collapsing of the walls commonly encountered during titania crystallization. The retention of the mesoporosity after thermal treatment clearly indicates the extremely high stability of these interpenetrating networks.

\section{Stability and crystal growth of anatase nanocrystals.}

Having elucidated the chemical structure and the stability of the mesostructure, the remaining question to be addressed was the evaluation of the crystal growth of anatase particles. In the whole and as expected, an increase in the crystal size is observed upon thermal treatment. However, compared to the literature results, the average size remains below $13 \mathrm{~nm}$, which is extremely interesting: the average particle size at $500^{\circ} \mathrm{C}$ is estimated to be $7 \mathrm{~nm}$ and up to $800^{\circ} \mathrm{C}$, the particle size does not exceed $11 \mathrm{~nm}$. In addition, no additional phase (brookite, rutile, titania phosphate) commonly obtained at $800^{\circ} \mathrm{C}$ can be detected herein witnessing the highest stability of this single phase heteroatom doped anatase (Figure 9 and Figure S11, Supporting information). Figure 10 clearly illustrates the virtues of these dendrimers in limiting the crystal growth of titanium dioxide particles, as the anatase generated with branchmimicking dendrimers (case of $\mathbf{M}_{\mathbf{4}}$ ) reaches already $12 \mathrm{~nm}$ at $500^{\circ} \mathrm{C}$ and even $24 \mathrm{~nm}$ at $800^{\circ} \mathrm{C}$ (Figure 10 ).

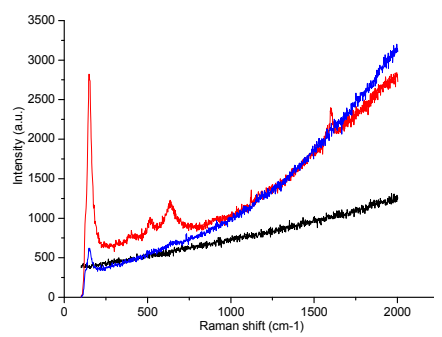

b)

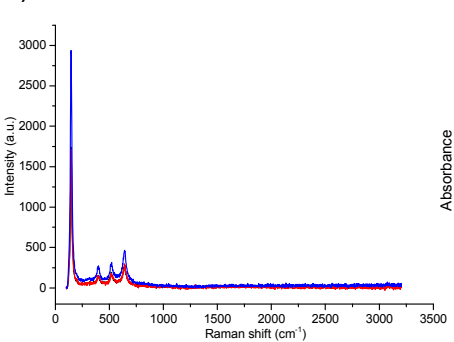

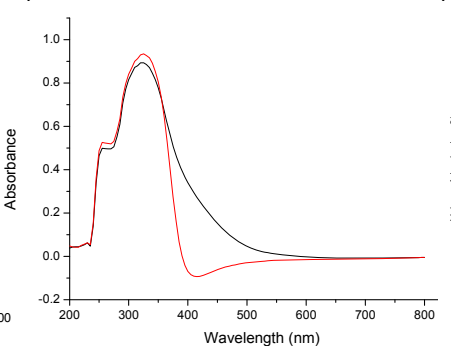

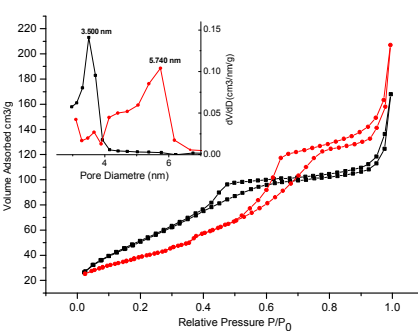

Fig. 8. a and b) RAMAN spectroscopic studies of the obtained materials a) before and d) after thermal annealing. After calcination, the anatase peaks become narrow and well resolved.c) UV-visible spectra of the tectonic materials before (in black) and after (in red) thermal annealing treatment. d) Nitrogen sorption analysis of the tectonic materials before (in black) and after (in red) thermal annealing treatment. Onset: The pore diameter distribution before and after thermal annealing treatment.
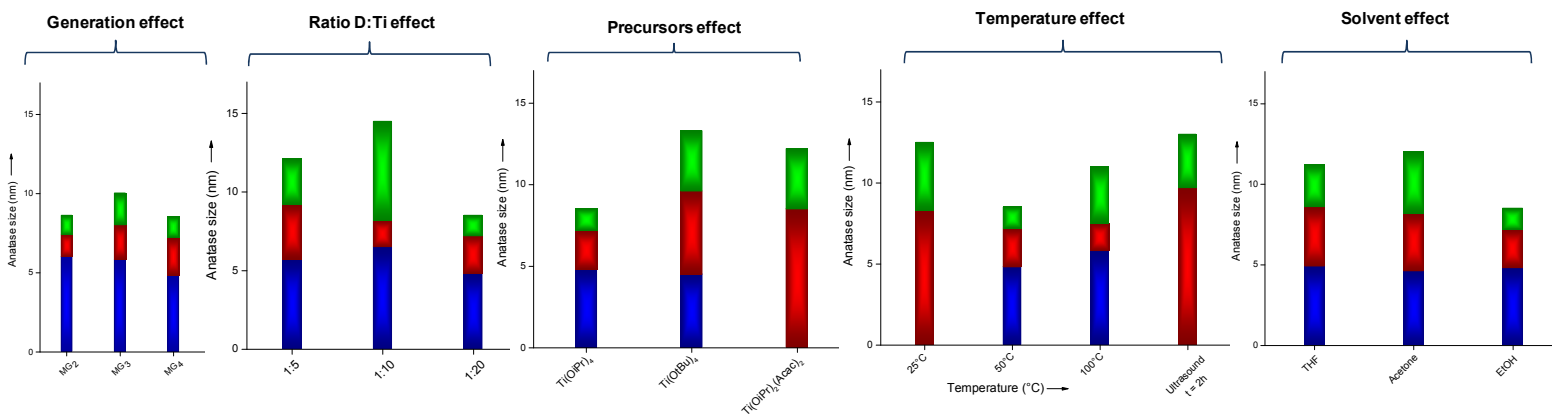

Fig. 9. Crystal size estimation of the generated anatase nanoparticles by X-ray diffraction (from Scherrer formula) as a function of several parameters. The maximum of the blue color corresponds to the crystal size at liquid temperature synthesis (unless specified at $\mathrm{T}=60^{\circ} \mathrm{C}$ ). The maximum of the red one corresponds to the size of the crystal obtained at $500^{\circ} \mathrm{C}$. The green level reflects the size of the crystal after thermal annealing at $800^{\circ} \mathrm{C}$. From left to right: dendrimer generation $\left(\mathbf{D G}_{2}, \mathbf{D G}_{3}\right.$ and $\left.\mathbf{D G}_{4}\right)$, respectively referred in Table 1 as $\mathbf{M}_{\mathbf{3}}, \mathbf{M}_{\mathbf{2}}$ and $\mathbf{M}_{\mathbf{1}}$. Ratio $\mathbf{D G}_{\mathbf{4}}: \mathrm{Ti}(\mathrm{OiPr})_{4}$ of 1:5, 1:10 and 1:20, respectively referred in Table 1 as $\mathbf{M}_{\mathbf{6}}, \mathbf{M}_{\mathbf{7}}$ and $\mathbf{M}_{\mathbf{1 0}}$. Precursor reactivity [Ti(OiPr) $)_{4}$, Ti(OtBu) $)_{4}$ and Ti(acac) $)_{2}(\mathrm{OiPr})_{2}$ ], respectively referred in Table 1 as $\mathbf{M}_{\mathbf{1}}, \mathbf{M}_{\mathbf{8}}$ and $\mathbf{M}_{\mathbf{9}}$. The Temperature $\left(25^{\circ} \mathrm{C}, 60^{\circ} \mathrm{C}, 100^{\circ} \mathrm{C}\right.$ and under ultrasonication for $\left.2 \mathrm{~h}\right)$, respectively referred in Table 1 as $\mathbf{M}_{\mathbf{1 1}}, \mathbf{M}_{\mathbf{1}}, \mathbf{M}_{\mathbf{1 0}}$ and $\mathbf{M}_{\mathbf{1 2}}$. The solvent nature (THF, acetone and ethanol), respectively referred in Table 1 as $\mathbf{M}_{14}, \mathbf{M}_{13}$ and $\mathbf{M}_{1}$.

The thermal annealing of the blank mineralization (preparation of $\mathbf{M}_{5}$ ), performed without any phosphorus additive, affords large anatase crystals $(27 \mathrm{~nm})$. Importantly, the cocondensation of branch-mimicking phosphonate, although less efficient in limiting the crystal size, proved however to be of interest to preserve the anatase phase up to $800^{\circ} \mathrm{C}$. In the absence any of phosphorus additives, the concomitant presence of rutile at $\mathrm{T}=800^{\circ} \mathrm{C}$ has been confirmed.

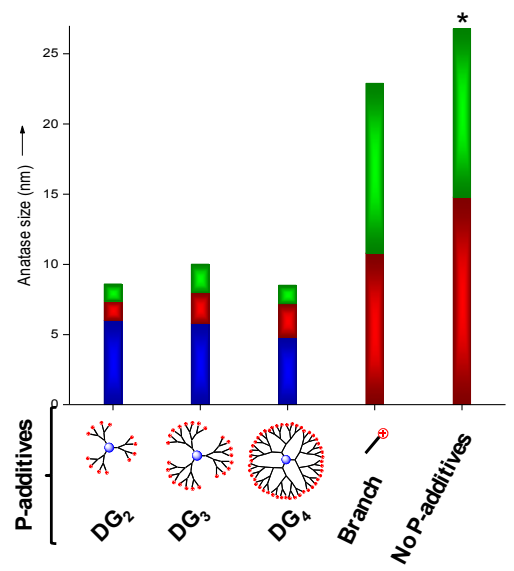


Fig. 10. Crystal growth of the anatase particles as a function of the phosphorus additive used. From left to right: second generation, third generation, fourth generation, branch-mimicking dendrimer and lastly the crystal growth without any phosphorus additive. The star indicates the presence of rutile at $800^{\circ} \mathrm{C}$. The maximum of the blue color corresponds to the size at $60^{\circ} \mathrm{C}$, the red level indicates the crystal size at $500^{\circ} \mathrm{C}$ and the green reflects the size of the crystal at $800^{\circ} \mathrm{C}$

Aiming to further elucidate the impact of the dendrimer medium on the expansion of the nanocrystals, the temperature-induced crystallization of the crystal grain size has been investigated for $\mathbf{M}_{\mathbf{1}}, \mathbf{M}_{\mathbf{4}}$ and $\mathbf{M}_{\mathbf{5}}$, obtained in presence of i) the fourth generation phosphorus dendrimer $\mathbf{D G}_{4}$, ii) the mimicked branch and iii) in native medium, without any phosphorus additive, respectively. The following equation (Equation. 1) was used:

$$
D^{2}(t, T)=D^{2}{ }_{0}+\text { k.t. } \exp \left(-E_{a} / R T\right) \quad \text { (Equation. 1) }
$$

Where $D_{0}$ is the initial grain size, $k$ is a temperatureindependent constant, $R$ is the gas constant and $E_{a}$ is the activation energy. ${ }^{41}$ In the presence of phosphorus dendrimer $\mathbf{D G}_{4}$, a lowest $E_{a}$ of $15.38 \mathrm{~kJ} \mathrm{~mol}^{-1}$ was obtained (Figure S12, Supporting Information). The activation energies calculated for the titanium mineralization using a branch-mimicking dendrimer $\left(\mathbf{M}_{\mathbf{4}}\right)$ or in the absence of any phosphorus additive $\left(\mathbf{M}_{5}\right)$, both of them have led to an amorphous titanium dioxide at $60^{\circ} \mathrm{C}$, were found to be respectively equal to 47.72 and 49.22 kJ.mol-1 (Figure S12, Supporting Information). The dramatic difference between the parent dendrimer and its branch fragment can be tentatively attributed to the presence of the crystal nucleus in the former solution synthesis, which acts probably as seeds for converting the remaining amorphous titania cross-linked to the dendrimer framework. Kohn et al. recently reported similar results. ${ }^{41}$ The value obtained herein $15.38 \mathrm{~kJ}^{\mathrm{mol}} \mathrm{m}^{-1}$ is much lower than that reported for anatase crystallization by Ostwald-ripening $\left(78 \mathrm{~kJ} \cdot \mathrm{mol}^{-1}\right){ }^{42}$ Owing to the sterical hindrance of the giant dendrimer, merging several amorphous particles to trigger conversion from amorphous titania to crystalline anatase must be also ruled out. The improved crystallization, implying marginal crystal growth, could be better explained by the crystallization of amorphous titania that are tightly connected to the anatase particles. The observed inhibition of the crystal expansion, naturally foreseen in the solid state, can be attributed to the formation of a phosphorus-rich glass phase that result from the thermal conversion of the dendrimer skeletal. This formed glass-like phase prevents the random coarsening of the nanocrystals and acts as a stabilizer for both crystal size and crystal phase. ${ }^{43}$

\section{Photo-activity for hydrogen generation}

Hydrogen production by water splitting is certainly one of the most exciting photo-catalytic reactions, principally for sustainable energy production from solar fuel. ${ }^{44}$ Considerable efforts were oriented to execute this reaction by means of nonmetallic activation, the most solicited catalyst being modified titania anatase. ${ }^{45}$ Considering the above-mentioned results in terms of heteroatom doping, improved crystallinity and mesoporosity, these materials seem to be ideal candidates. Beside our curiosity to assess their photo-catalytic activity, we were primarily interested to assess the difference in improving crystallinity (as synthesized versus thermally annealed) as well as the effect of increasing heteroatom content $(P, N)$ by increasing the dendrimer generation. ${ }^{31}$ With these two goals in mind, we embarked for hydrogen production using for titaniabased catalyst: the hybrid material $\mathbf{M}_{2}$ obtained from the $D_{3}$ with ethanol as solvent at $60^{\circ} \mathrm{C}$ (Table 1), its thermally-annealed version referred as $\mathbf{M}_{\mathbf{2}}-\mathbf{5 0 0}$ and two other calcined materials drived from $\mathbf{D G}_{\mathbf{2}}$ and $\mathbf{D G}_{\mathbf{4}}$ phosphorus dendrimers (namely $\mathbf{M}_{\mathbf{3}}$ 500 and $\mathbf{M}_{\mathbf{1}}-\mathbf{5 0 0}$, respectively) (See S1e in SI). Indeed, while the non-calcined material $\mathbf{M}_{\mathbf{2}}$ showed the highest adsorption in the visible region, hydrogen generated by its use remains poor. In contrast, its thermally treated analogue $\mathbf{M}_{\mathbf{2}}-\mathbf{5 0 0}$, although absorbing only in UV-region, displayed greatest catalytic activity (Figure 11, right). Thermal treatment converts inactive amorphous titania to highly reactive anatase without significant expansion of the crystal size (crystal size of the anatase is 5.8 $\mathrm{nm}$ for the as-synthesized material versus $8 \mathrm{~nm}$ for the calcined one). This comparison indicates the importance of improving the crystallinity of the material framework. Excitingly, the comparison of thermally annealed materials with increasing content of heteroatoms indicates an increase of the photocatalytic activity of tested titania with increasing dendrimer generation, with the material $\mathbf{M}_{\mathbf{1}} \mathbf{- 5 0 0}$ drived from the use of $\mathbf{D G}_{4}$ being the most active one (Figure 11, left). Hydrogen production increases linearly with time, meaning that no significant deactivation of the catalyst occurs.
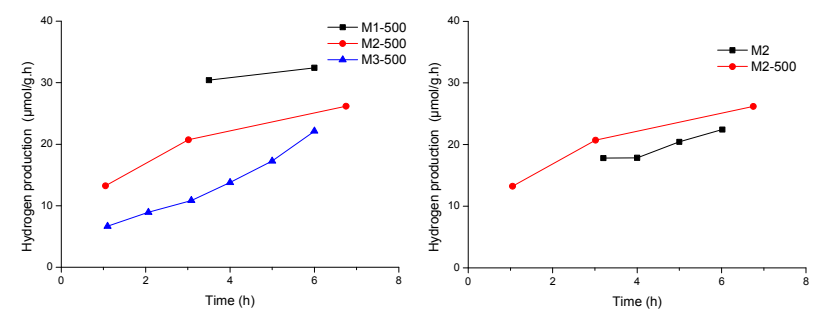

Fig. 11. Photo-catalytic hydrogen evolution by water splitting. Left: effect of dendrimer generation heteroatom doping. Right: effect of thermal treatment.

\section{Conclusion}

Since the infancy of the sol-gel mineralization of molecular titanium alkoxide species, it has been assumed that only amorphous titanium dioxide phase can be obtained at synthetic medium, low temperature synthesis. The requested crystalline anatase was primarily generated under thermal annealing treatment at high temperatures, generally exceeding $500^{\circ} \mathrm{C}$. Strikingly, for more than a decade, this thorny issue has not been tackled and the general scheme of titanium mineralization described in classical textbooks (amorphous state ---> crystalline anatase ---> rutile and/or brookite) has been believed to be thermodynamically unavoidable. The ability to generate crystalline anatase at lower temperature, thereby escaping the harsh thermal treatment, is of paramount interest in the field of hybrid material synthesis. Processing at lower temperature can keep intact the organic part of the material (for instance, photosensitizers in DSSC) and allow depositing the crystalline material on less-expensive, organic (flexible or rigid) substrates. Hitherto, the implementation of these cost-effective substrates in the conception of energetic devices is hindered by their limited stability, being non-suitable for processing above $180^{\circ} \mathrm{C}$ at most. The questionable ability of titanium-oxo-species to undergo crystallization at low temperature synthesis has been recently validated under non-conventional synthetic route: ex. Ionic liquids, benzyl alcohol and microwave heating. ${ }^{12-}$ 14 Boosted by these precedents, mineralization of titanium 
alkoxide is performed herein in presence of phosphonateterminated dendrimers. This strategy affords discrete crystalline anatase nanoparticles. The "particularism" of the used phosphorus-dendrimers is illustrated by their marked difference to their branch analogues, which led only amorphous titanium dioxide. The mandatory presence of phosphorus dendrimers in the sol-gel medium originates from their unique architecture: beside their swelling behavior that generate an open porous framework, the presence of two antagonisitic functions within the dendrimer skeletal (hydrophobic cyclotriphosphazene core and polar ionic phosphonic surface) are at the basis of their amphiphilic character. This allows to drive water and polar solvent/monomers to the interface periphery and thus to increase the concentration of the sol-gel reactants by compartmentalization. The contrasted behavior of the phosphorus dendrimers versus their branch-mimicking analogues is reminiscent to the difference in the supramolecular assembly encountered depending on the critical micelle concentration (isolated single molecules below $\mathrm{CMC}$ versus self-assembled nanoreactors above $\mathrm{CMC}$ ). Noteworthy herein is the $60^{\circ} \mathrm{C}$ synthesis temperature, which is, to the best of our knowledge, one of the lowest temperatures reported to date for anatase crystallization. An exhaustive investigation on diverse sol-gel parameters (solvent nature, temperature, dendrimer-to-metal ratio, sol-gel precursor reactivity) reveals many informative results in terms of the crystallinity and the mesoporosity. For instance, the sol-gel mineralization performed using the less-reactive $\mathrm{Ti}(\mathrm{acac})_{2}(\mathrm{OiPr})_{2}$ (synthesis of $\mathbf{M}_{\mathbf{g}}$ ) as well as the sol-gel performed at a lowest temperature of $25^{\circ} \mathrm{C}$ (synthesis of $\mathbf{M}_{11}$ and $\mathbf{M}_{12}$ ) failed to generate crystalline anatase. In these two cases, the network is found to be less condensed as revealed by TGA. Indeed, while the presence of phosphonate-terminated dendrimers is mandatory for low temperature crystallization, an energy input is required to allow sufficient nucleation and thus a critical matter condensation. This last point is revealed herein of paramount interest; the low condensation of the nucleated network constitutes an impediment for titanium dioxide crystallization. The diversity of heteroatoms in the dendrimer skeletal (from the core to the branch to those in the external surface) allows a temperature-dependent, continuous delivering of heteroatoms to the boundaries of the growing crystal upon heat treatment. The sterical hindrance of these dendrimers and the heteroatom doping to the titania surfaces are the basis for restricting the crystal growth to $7 \mathrm{~nm}$ up to $500^{\circ} \mathrm{C}$ and $13 \mathrm{~nm}$ at up to $800^{\circ} \mathrm{C}$ and accounts for preserving its mesoporosity. The unusual properties of the used phosphonate-terminated phosphorus dendrimers allows for extending the broad temperature stability of the generated anatase phase (from $60^{\circ} \mathrm{C}$ to $800^{\circ} \mathrm{C}$ ) with no formation of brookite or rutile. Increasing the dendrimer generation (thereby increasing the percent of heteroatom doping within the mineral phase) provides the driving force for enhancing their photocatalytic water-splitting. Work is in progress to gain better understanding on their structure-reactivity relationship.

\section{Notes and references}

¥ Footnotes relating to the main text should appear here. These might include comments relevant to but not central to the matter under discussion, limited experimental and spectral data, and crystallographic data.

1 (a) F. Hoffmann, M. Cornelius, J. Morell and M. Fröba, Angew. Chem. Int. Ed., 2006, 45, 3216-3251; (b) J. L. Townson, Y.-S. Lin, J. O. Agola, E. C. Carnes, H. S. Leong, J. D. Lewis, C. L. Haynes and C. J. Brinker, J. Am. Chem. Soc., 2013, 135, 16030-16033.

2 L. Nicole, C. Laberty-Robert, L. Rozes and C. Sanchez, Nanoscale, 2014, 6, 6267-6292.

3 Chem. Soc. Rev., 2011, 40, 453 - 1152; themed issue on hybrid materials

4 (a) C. J. Brinker and G. W. Scherer, Sol-gel Science: The Physics and Chemistry of Sol-gel Processing, Academic Press, 1990; (b) H. E. Bergna and W. O. Roberts, Colloidal silica fundamentals and applications, Taylor and Francis, Boca Raton, Fla.; London; New York, 2006.

5 (a) M. Ide, E. De Canck, I. Van Driessche, F. Lynen and P. Van Der Voort, RSC Adv., 2015, 5, 5546-5552; (b) M. I. López, D. Esquivel, C. Jiménez-Sanchidrián, P. Van Der Voort and F. J. RomeroSalguero, J. Phys. Chem. C, 2014, 118, 17862-17869.

6 (a) G. Guerrero, J. G. Alauzun, M. Granier, D. Laurencin and P. H. Mutin, Dalton Trans., 2013, 42, 12569-12585; (b) P. H. Mutin, G. Guerrero and A. Vioux, C. R. Chim., 2003, 6, 1153-1164; (c) P. H. Mutin, G. Guerrero and A. Vioux, J. Mater. Chem., 2005, 15, 37613768; (d) R. Boissezon, J. Muller, V. Beaugeard, S. Monge and J.-J. Robin, RSC Adv., 2014, 4, 35690-35707. (e) C. Queffélec, M. Petit, P. Janvier, D. A. Knight and B. Bujoli, Chem. Rev., 2012, 112, 37773807.

7 (a) G. Guerrero, P. H. Mutin and A. Vioux, Chem. Mater., 2000 12, 1268-1272; (b) T.-Y. Ma and Z.-Y. Yuan, ChemSusChem, 2011, 4, 1407-1419; (c) Y. Brahmi, N. Katir, A. Hameau, A. Essoumhi, E. M. Essassi, A.-M. Caminade, M. Bousmina, J.-P. Majoral and A. EI Kadib, Chem. Commun., 2011, 47, 8626-8628; (d) Y. Brahmi, N. Katir, M. lanchuk, V. Colliere, E. M. Essassi, A. Ouali, A.-M. Caminade, M. Bousmina, J. P. Majoral and A. El Kadib, Nanoscale, 2013, 5, 2850-2856; (e) T.-Y. Ma, X.-Z. Lin and Z.-Y. Yuan, J. Mater. Chem., 2010, 20, 7406-7415; (f) Y.-P. Zhu, T.-Y. Ma, Y.-L. Liu, T.-Z. Ren and Z.-Y. Yuan, Inorg. Chem. Front., 2014, 1, 360-383; (g) A. Dutta, A. K. Patra, S. Dutta, B. Saha and A. Bhaumik, J. Mater. Chem., 2012, 22, 14094-14100.

8 (a) X.-J. Zhang, T.-Yi Ma and Z.-Y. Yuan, J. Mater. Chem., 2008, 18, 2003-2010; (b) M. Pramanik, A. K. Patra and A. Bhaumik Dalton Trans., 2013, 42, 5140-5149

9 (a) S. Y. Choi, M. Mamak, N. Coombs, N. Chopra and G. A. Ozin, Nano Lett., 2004, 4, 1231-1235; (b) Y. Amao, Curr. Nanosci., 2008, 4, 45-52; (c) X. Marguerettaz, G. Redmond, S. Nagaraja Rao and D. Fitzmaurice, Chem. Eur. J., 1996, 2, 420-428. (d) Y. P. Zhu, T. Y. Ma, T. Z. Ren, J. Li, G. H. Du, Z. Y. Yuan. Appl. Catal. B. Env., 2014, 156-157, 44-52.

10 (a) X. Chen and S. S. Mao, Chem. Rev., 2007, 107, 2891-2959; (b) D. Fattakhova-Rohlfing, A. Zaleska and T. Bein, Chem. Rev., 2014, 114, 9487-9558.

11 J. N. Kondo and K. Domen, Chem. Mater., 2007, 20, 835-847.

12 (a) Y. Zhou and M. Antonietti, J. Am. Chem. Soc., 2003, 125, 14960-14961; (b) T. Nakashima and N. Kimizuka, J. Am. Chem. Soc., 2003, 125, 6386-6387; (c) K. Ding, Z. Miao, B. Hu, G. An, Z. Sun, B. Han and Z. Liu, Langmuir, 2009, 26, 5129-5134.

13 (a) M. Niederberger, M. H. Bartl and G. D. Stucky, J. Am. Chem. Soc., 2002, 124, 13642-13643; (b) M. Niederberger, M. H. Bartl and G. D. Stucky, Chem. Mater., 2002, 14, 4364-4370.

14 B. Reeja-Jayan, K. L. Harrison, K. Yang, C.-L. Wang, A. E. Yilmaz and A. Manthiram, Sci. Rep., 2012, 2.

15 M. Pini, R. Rosa, P. Neri, F. Bondioli and A. M. Ferrari, Green Chem., 2015, 17, 518-531.

16 (a) Y. Noda, B. Lee, K. Domen and J. N. Kondo, Chem. Mater., 2008, 20, 5361-5367; (b) J. Lee, M. Christopher Orilall, S. C. Warren, M. Kamperman, F. J. DiSalvo and U. Wiesner, Nat. Mater., 2008, 7, 222-228. 
17 J. M. Szeifert, D. Fattakhova-Rohlfing, D. Georgiadou, V Kalousek, J. Rathouský, D. Kuang, S. Wenger, S. M. Zakeeruddin, M. Grätzel and T. Bein, Chem. Mater., 2009, 21, 1260-1265. 18 D. Li, H. Zhou and I. Honma, Nat. Mater., 2004, 3, 65-72.

19 (a) W. Zhou, F. Sun, K. Pan, G. Tian, B. Jiang, Z. Ren, C. Tian and H. Fu, Adv. Funct. Mater., 2011, 21, 1922-1930; (b) G. Tian, H. Fu, L. Jing, B. Xin and K. Pan, J. Phys. Chem. C, 2008, 112, 3083-3089. 20 B. Tian, X. Liu, B. Tu, C. Yu, J. Fan, L. Wang, S. Xie, G. D. Stucky and D. Zhao, Nat. Mater., 2003, 2, 159-163.

21 (a) R. Buonsanti, T. E. Pick, N. Krins, T. J. Richardson, B. A. Helms and D. J. Milliron, Nano Lett., 2012, 12, 3872-3877; (b) I. E. Rauda, R. Buonsanti, L. C. Saldarriaga-Lopez, K. Benjauthrit, L. T. Schelhas, M. Stefik, V. Augustyn, J. Ko, B. Dunn, U. Wiesner, D. J. Milliron and S. H. Tolbert, ACS Nano, 2012, 6, 6386-6399.

22 D. A. Tomalia, H. Baker, J. Dewald, M. Hall, G. Kallos, S. Martin, J. Roeck, J. Ryder and P. Smith, Macromolecules, 1986, 19, 24662468.

23 (a) N. Satoh, T. Nakashima, K. Kamikura and K. Yamamoto, Nat. Nano., 2008, 3, 106-111; (b) G. J. d. A. A. Soler-lllia, L. Rozes, M. K. Boggiano, C. Sanchez, C.-O. Turrin, A.-M. Caminade and J.-P. Majoral, Angew. Chem. Int. Ed., 2000, 39, 4249-4254; (c) S. L. Sewell, R. D. Rutledge and D. W. Wright, Dalton Trans., 2008, 3857-3865.

24 N. Katir, A. El Kadib, V. Colliere, J. P. Majoral and M. Bousmina, Chem. Commun., 2014, 50, 6981-6983.

25 A. El Kadib, N. Katir, M. Bousmina and J. P. Majoral, New J. Chem., 2012, 36, 241-255.

26 C.-O. Turrin, V. Maraval, J. Leclaire, E. Dantras, C. Lacabanne, A.-M. Caminade and J.-P. Majoral, Tetrahedron, 2003, 59, 39653973.

27 R. Asahi, T. Morikawa, H. Irie and T. Ohwaki, Chem. Rev., 2014, 114, 9824-9852.

28 (a) A. E. Kadib, K. Molvinger, T. Cacciaguerra, M. Bousmina and D. Brunel, Microporous Mesoporous Mater., 2011, 142, 301-307; (b) A. El Kadib and M. Bousmina, Chem. Eur. J., 2012, 18, 82648277; (c) W. Li, Z. Wu, J. Wang, A. A. Elzatahry and D. Zhao, Chem. Mater., 2013, 26, 287-298; (d) U. Schubert, J. Mater. Chem., 2005, 15, 3701-3715

29 (a) L. Rozes and C. Sanchez, Chem. Soc. Rev., 2011, 40, 10061030; (b) F. Perineau, S. Pensec, C. Sanchez, C. Creton, L. Rozes and L. Bouteiller, Polym. Chem., 2011, 2, 2785-2788.

30 (a) C. Maurer, E. Pittenauer, V. A. Du, G. Allmaier and U. Schubert, Dalton Trans., 2012, 41, 2346-2353; (b) U. Schubert, Chem. Soc. Rev., 2011, 40, 575-582; (c) M. Lomoschitz, H. Peterlik, K. Zorn, S. O. Baumann and U. Schubert, J. Mater. Chem., 2010, 20, 5527-5532; (d) M. Puchberger, W. Rupp, U. Bauer and U. Schubert, New J. Chem., 2004, 28, 1289-1294.

31 A.-M. Caminade, A. Ouali, R. Laurent, C.-O. Turrin and J.-P. Majoral, Chem.Soc. Rev., 2015,44, 3890-3899.

32 (a) A.-M. Caminade and J.-P. Majoral, J. Mater. Chem., 2005, 15, 3643-3649; (b) A.-M. Caminade, B. Delavaux-Nicot and J.-P. Majoral in Hybrid Materials Built from (Phosphorus) Dendrimers, Vol. John Wiley \& Sons, Inc., 2014, pp. 503-525.

33 (a) C. Sanchez, J. Livage, M. Henry and F. Babonneau, J. NonCryst. Solid., 1988, 100, 65-76; (b) A. Leaustic, F. Babonneau and J. Livage, Chem. Mater., 1989, 1, 240-247; (c) A. Leaustic, F. Babonneau and J. Livage, Chem. Mater., 1989, 1, 248-252; (d) A E. Kadib, K. Molvinger, C. Guimon, F. Quignard and D. Brunel, Chem. Mater., 2008, 20, 2198-2204.

34 (a) J. Leclaire, Y. Coppel, A.-M. Caminade and J.-P. Majoral, J. Am. Chem. Soc., 2004, 126, 2304-2305; (b) J. Jiménez, A. Laguna, A. M. Molter, J. L. Serrano, J. Barberá and L. Oriol, Chem. Eur. J., 2011, 17, 1029-1039; (c) P. Carbone and L. Lue, Macromolecules, 2010, 43, 9191-9197; (d) D. Astruc, E. Boisselier and C. Ornelas, Chem. Rev., 2010, 110, 1857-1959; (e) R. M. Crooks, M. Zhao, L. Sun, V. Chechik and L. K. Yeung, Acc. Chem. Res., 2000, 34, 181190.

35 K. J. Shea and D. A. Loy, Chem. Mater., 2001, 13, 3306-3319.
36 A. El Kadib, P. Hesemann, K. Molvinger, J. Brandner, C. Biolley, P. Gaveau, J. J. E. Moreau and D. Brunel, J. Am. Chem. Soc., 2009, 131, 2882-2892.

37 Y. Wang, T. Sun, D. Yang, H. Liu, H. Zhang, X. Yao and H. Zhao, Phys. Chem. Chem. Phys., 2012, 14, 2333-2338.

38 (a) T.-Y. Ma, X.-Z. Lin and Z.-Y. Yuan, Chem. Eur. J., 2010, 16, 8487-8494; (b) J. El Haskouri, C. Guillem, J. Latorre, A. Beltrán, D. Beltrán and P. Amorós, Chem. Mater., 2004, 16, 4359-4372; (c) M. Gleria and R. De Jaeger, Phosphazenes: A Worldwide Insight, Nova Science Publishers, 2004.

39 R. Zheng, L. Lin, J. Xie, Y. Zhu and Y. Xie, J. Phys. Chem. C, 2008, 112, 15502-15509.

40 P. Periyat, N. Leyland, D. E. McCormack, J. Colreavy, D. Corr and S. C. Pillai, J. Mater. Chem., 2010, 20, 3650-3655.

41 P. Kohn, S. Pathak, M. Stefik, C. Ducati, U. Wiesner, U. Steiner and S. Guldin, Nanoscale, 2013, 5, 10518-10524 42 H. Zhang and J. F. Banfield, Chem. Mater., 2002, 14, 4145-4154. 43 P. H. Gaskell, J. Non-Cryst. Solid, 1995, 192-193, 9-22.

44 (a) T. J. Meyer, Acc. Chem. Res., 1989, 22, 163-170; (b) D. Gust, T. A. Moore and A. L. Moore, Acc. Chem. Res., 2009, 42, 18901898.

45 (a) M. R. Hoffmann, S. T. Martin, W. Choi and D. W. Bahnemann, Chem. Rev., 1995, 95, 69-96; (b) A. L. Linsebigler, G. Lu and J. T. Yates, Chem. Rev., 1995, 95, 735-758; (c) M. A. Fox and M. T. Dulay, Chem. Rev., 1993, 93, 341-357. 